\title{
XMM-Newton observation of the distant $(z=0.6)$ galaxy cluster RX J1120.1+4318
}

\author{
M. Arnaud ${ }^{1}$, S. Majerowicz 1 , D. Lumb ${ }^{2}$, D. M. Neumann ${ }^{1}$, N. Aghanim ${ }^{3}$, A. Blanchard ${ }^{4}$, M. Boer ${ }^{5}$, D. J. Burke ${ }^{6}$, \\ C. A. Collins ${ }^{7}$, M. Giard ${ }^{5}$, J. Nevalainen ${ }^{8,9}$, R. C. Nichol $^{10}$, A. K. Romer ${ }^{10}$, and R. Sadat ${ }^{5}$
}

${ }^{1}$ CEA/DSM/DAPNIA, Service d'Astrophysique, L'Orme des Merisiers, Bât. 709, 91191 Gif-sur-Yvette, France

2 Science Payloads Technology Divn., Research and Science Support Dept., European Space Agency, ESTEC, Keplerlaan 1, Postbus 299, 2200AG Noordwijk, The Netherlands

3 Institut d'Astrophysique Spatiale, Université Paris-Sud, 91405 Orsay Cedex, France

${ }^{4}$ Laboratoire d'astrophysique de l'Observatoire Midi-Pyrénées, UMR5572, CNRS, UPS, 14 Av. E. Belin, 31400 Toulouse, France

5 Centre d'Etude Spatiale des Rayonnements, 9 avenue du colonel Roche, BP 4346, 31028 Toulouse, France

${ }^{6}$ Harvard-Smithsonian Center for Astrophysics, 60 Garden Street, Cambridge, MA 02138, USA

7 Astrophysics Research Institute, Liverpool John Moores University, Twelve Quays House, Egerton Wharf, Birkenhead L41 1LD, UK

${ }^{8}$ European Space Agency, Research and Scientific Support Division, ESTEC, Postbus 299 Keplerlaan 1, 2200AG Noordwijk, The Netherlands

9 Present adress: University of Alabama in Huntsville, Physics Department, OB 201B Huntsville, AL 35899, USA

${ }^{10}$ Physics Department, Carnegie Mellon University, 5000 Forbes Avenue, Pittsburgh, PA15213, USA

Received 3 April 2002 / Accepted 24 April 2002

\begin{abstract}
We report on a $20 \mathrm{ks}$ XMM observation of the distant cluster RX J1120.1+4318, discovered at $z=0.6$ in the SHARC survey. The cluster has a regular spherical morphology, suggesting it is in a relaxed state. The combined fit of the EPIC/MOS\&pn camera gives a cluster mean temperature of $k T=5.3 \pm 0.5 \mathrm{keV}$ with an iron abundance of $0.47 \pm 0.19$. The temperature profile, measured for the first time at such a redshift, is consistent with an isothermal atmosphere up to half the virial radius. The surface brightness profile, measured nearly up to the virial radius, is well fitted by a $\beta$-model, with $\beta=0.78_{-0.04}^{+0.06}$ and a core radius of $\theta_{\mathrm{c}}=0.44_{-0.04}^{+0.06} \mathrm{arcmin}$. We compared the properties of RX J1120.1+4318 with the properties of nearby clusters for two cosmological models: an Einstein-de Sitter Universe and a flat low density Universe with $\Omega_{0}=0.3$. For both models, the scaled emission measure profile beyond the core, the gas mass fraction and luminosity are consistent with the expectations of the self-similar model of cluster formation, although a slightly better agreement is obtained for a low density Universe. There is no evidence of a central cooling flow, in spite of the apparent relaxed state of the cluster. This is consistent with its estimated cooling time, larger than the age of the Universe at the cluster redshift. The entropy profile shows a flat core with a central entropy of $\sim 140 \mathrm{keV} \mathrm{cm}$, remarkably similar to the entropy floor observed in nearby clusters, and a rising profile beyond typically 0.1 virial radius. Implications of our results, in terms of non-gravitational physics in cluster formation, are discussed.
\end{abstract}

Key words. galaxies: clusters: general - galaxies: intergalactic medium - cosmology: observations - cosmology: dark matter - cosmological parameters - X-rays: galaxies: clusters

\section{Introduction}

Galaxy clusters occupy a unique position in the scenario of hierarchical structure formation, as they are still forming today. The statistical properties of this evolving population (e.g. the mass distribution function and the correlations between physical quantities, at various redshifts) therefore provide unique constraints on cosmological scenarios.

In the simplest model of structure formation, purely based on gravitation, galaxy clusters constitute a homologous

Send offprint requests to: M. Arnaud,

e-mail: marnaud@discovery.saclay.cea.fr family. Clusters are self-similar in shape, and predictable scaling laws relate each physical property to the cluster total mass (or temperature) and redshift $z$ (e.g. Bryan \& Norman 1998; Eke et al. 1998). From observations with ROSAT and ASCA satellites, it is now well established that this simple model fails to explain all the observed structural and scaling properties of the nearby cluster population (Tozzi \& Norman 2001 and reference therein). The evolution with redshift of these properties is an essential piece of information, still largely missing, to reconstruct the physics of the formation process. This information is also important for accurate $\Omega_{0}$ estimates based on the evolution of the cluster mass function (e.g. Oukbir \& Blanchard 1997). 
To compare the results of flux-limited X-ray surveys with the prediction of the various theoretical models, it is necessary to understand the relation between observed quantities, like the cluster temperature and luminosity, and the virial mass. Precise $\Omega_{0}$ estimate also requires a good understanding of the survey selection function, which further depends sensitively on cluster morphology (e.g. Adami et al. 2000).

Following the pioneering work of the Einstein Medium Sensitivity Survey (Gioia et al. 1990), several large, well controlled, X-ray samples of distant clusters have been assembled in the last years, using ROSAT observations (see Gioia 2000 for a review). The exceptional sensitivity of XMM, associated with good spectroscopic and imaging capabilities, now allows the detailed analysis of these clusters, down to what was only a detection limit with ROSAT. We present here the XMM observation of RX J1120.1+4318, a cluster at $z=0.6$ detected in the bright Serendipitous High-redshift Archival ROSAT Cluster (SHARC) survey (Romer et al. 2000). This observation was made in the framework of the XMM-Newton $\Omega$ project, a systematic XMM Guaranteed Time follow-up of the most distant $(z>0.45)$ SHARC clusters (Bartlett et al. 2001).

The paper is organized as follows. In Sect. 2, we describe the data analysis performed to derive the surface brightness profile, the global temperature and the temperature profile, which are presented in Sect. 3. In Sect. 4, we compare the physical properties of RX J1120.1+4318 with the predictions of the self-similar model of cluster formation. The scaled emission measure and temperature profiles, the gas mass fraction and $L_{\mathrm{X}}-T$ relation are compared to those of nearby clusters. This study is made for two cosmological models: an Einsteinde Sitter Universe $\left(\mathrm{EdS}, \Omega_{0}=1\right)$ and a flat low density Universe $(\Lambda \mathrm{CDM})$ with $\Omega_{0}=0.3$ and $\Lambda=0.7$. In Sect. 5, we study the thermodynamical state of this cluster - possible presence of cooling gas, entropy content - to further assess the role of non-gravitational processes in cluster formation. Section 6 contains our conclusions.

Unless otherwise stated all errors on the cluster parameters are at the $90 \%$ confidence level. A Hubble constant of $H_{0}=$ $50 \mathrm{~km} \mathrm{~s}^{-1} \mathrm{Mpc}^{-1}$ is assumed. At the cluster redshift, 1 arcmin corresponds to $456 \mathrm{kpc}$ and $561 \mathrm{kpc}$ for the EdS model and the $\Lambda \mathrm{CDM}$ model, respectively.

\section{Data analysis}

\subsection{Data preparation}

RX J1120.1+4318 was observed for 20 ks on May 8, 2001 with the EPIC/MOS and pn camera (using the THIN optical blocking filter) in Full Frame mode (Turner et al. 2001; Strüder et al. 2001). We generated calibrated event files using the tasks emchain and epchain of the SAS V5.1.

We discarded the data corresponding to the periods of high background induced by solar flares (e.g. see Arnaud et al. 2001). We extracted the light curves in the energy band [10-12] keV and [12-14] keV for the MOS and pn data respectively. In these energy bands, the effective area of XMM is negligible and the emission is dominated by the particle induced background. We removed all frames corresponding to a count rate greater than $15 \mathrm{ct} / 100 \mathrm{~s}$ (MOS data) and $22 \mathrm{ct} / 100 \mathrm{~s}$ (pn data). After this selection, the remaining exposure time is $17.6 \mathrm{ks}, 17.9 \mathrm{ks}$ and $14.2 \mathrm{ks}$ for the MOS1, MOS2 and pn observations.

\subsection{Vignetting correction}

The effective area of the XMM mirrors is a function of off-axis angle and this vignetting effect depends on energy. An additional vignetting effect is due to the RGA obscuration for the MOS camera. The vignetting calibration data were those available at time of release of SAS V5.1.

To correct for vignetting effects, we used the method proposed by Arnaud et al. (2001) for spectra, which can be generalized in a straightforward way to profiles or images (e.g. Majerowicz et al. 2002). For each event, we computed the corresponding weight coefficient, defined as the ratio of the effective area at the photon position and energy to the central effective area at that energy. When extracting spectra, image or surface brightness profile, each event is weighted by this coefficient ${ }^{1}$. These "corrected" products correspond to those we would obtain if the detector response were flat. The on-axis response can then be used for spectral fitting and to estimate the physical parameters of the cluster. We used the spectral response files m1_thin1v9q20t5r6_all_15.rsp, m2_thin1v9q20t5r6_all_15.rsp for EPOC/MOS1,2 and epn_ff20_sY9_thin.rmf V6.1 for EPIC/pn respectively ${ }^{2}$. Those files include both the effective area and the redistribution matrix.

\subsection{Background subtraction}

After cleaning for flare events, the XMM background is dominated by the Cosmic X-ray background (CXB) and the non $\mathrm{X}$ ray background (NXB) induced by high energy particles. The former component, which dominates at low energy, depends on the observed position in the sky and is vignetted by the optics. The latter component, which dominates at high energy, is not vignetted but varies slightly across the detector and with time.

To subtract the total XMM background, we used EPIC blank sky event files (one for each camera) obtained by combining several high galactic latitude pointings. The data are cleaned for background flares and bright point sources are excluded (Lumb 2002). The sky coordinates in the event files were modified using the aspect solution of the RX J1120.1+4318 observation, so that extraction can be done in sky coordinates, while insuring that the same detector region is considered for both the blank field and the source observation. The background level, estimated from the total count rate in the whole FOV in the high energy bands defined above, was found to be 0.85 times smaller for the source observation than for the blank sky data. This is typical of the variations observed since launch (Lumb 2002), which are explainable by variations in Cosmic Ray shielding as the magnetosphere is

\footnotetext{
1 This vignetting correction can now be done with task EVIGWEIGHT of SAS V5.2.

2 see http://xmm.vilspa.esa.es/calibration/
} 


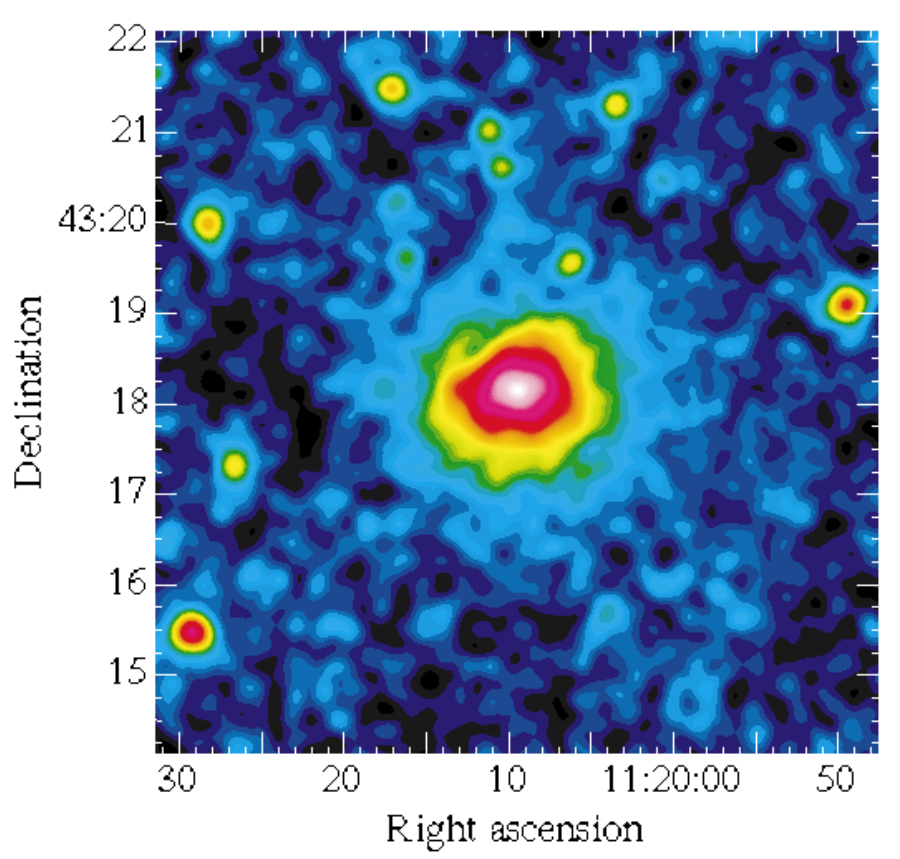

Fig. 1. Combined EPIC/MOS1\&MOS2\&pn image of RX J1120.1+ 4318 in the [0.3-5] keV energy band (linear intensity).

pumped up and down by solar activity. The background subtraction for each source product (spectrum or profile) is done in two steps. The method is fully described in Appendix A. We first subtract the corresponding blank field product, obtained using the same spatial and energy selection, and normalized by the 0.85 factor defined above. For consistency, the blank field products were obtained using the same vignetting correction method as that used for the source. The correction factor is thus formally wrong for the NXB component. However, as we subtract blank field and source count rates estimated in the same region in detector coordinates this correction factor is the same and does not introduce bias. This first step thus allows us to subtract properly the NXB. However, we are left with a residual $\mathrm{CXB}$ component, which is the difference between the CXB in the source region and in the blank fields (multiplied by the normalization factor above). This residual is corrected for vignetting and is expected to be uniform all over the field of view. In a second step, we thus subtract this residual component, using data in the outer part of the FOV, outside the cluster region.

\section{Results}

The vignetting corrected image in the [0.3-5] keV energy band is displayed in Fig. 1. The data of all EPIC cameras are combined. The cluster has a regular spherical morphology, suggesting it is in a relaxed state.

\subsection{Surface brightness profile}

We extracted the surface brightness profile of the cluster in the [0.3-3] keV energy band. This band was chosen to optimize the signal-to-noise $(S / N)$ ratio. We cut out serendipitous sources in the field of view and binned the photons into con-

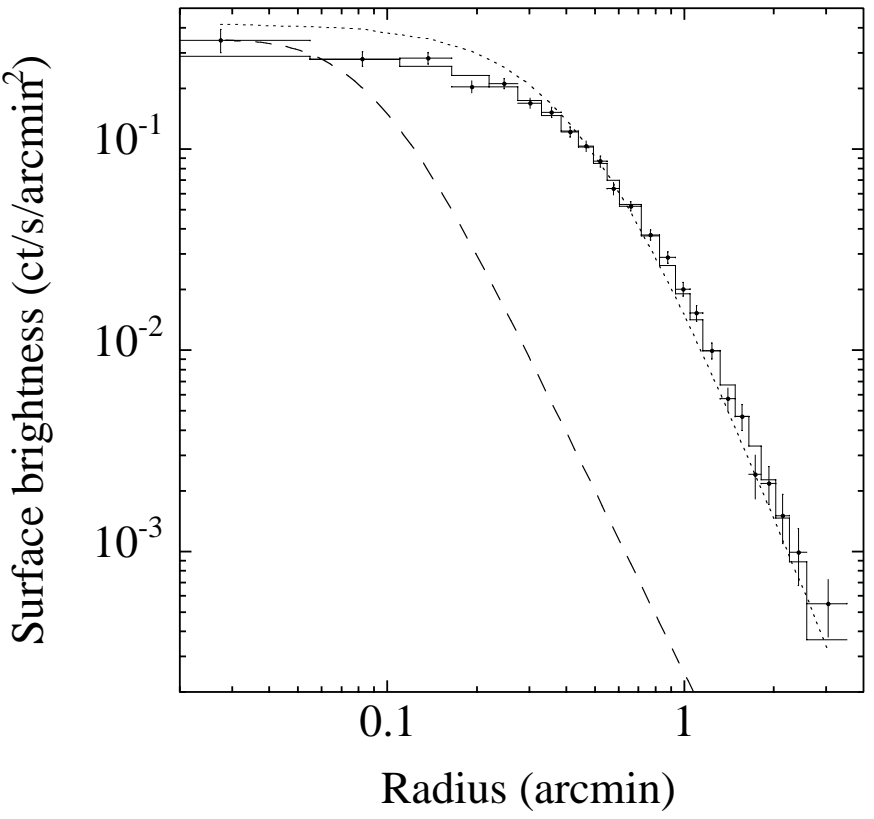

Fig. 2. Combined MOS1, MOS2 and pn surface brightness profile RX J1120.1+4318 in the [0.3-3] keV energy band. Dotted line: best fit $\beta$-model. Solid line: same convolved with the XMM/PSF and binned as the data. Dashed line: on axis XMM/PSF, normalized to the central intensity.

centric annuli with a width of 3.3" (3 pixels of the MOS camera) centered on the maximum of the X-ray emission for each camera. Since the cluster is regular, this center corresponds to the centroid of the emission. The three profiles were then summed. The vignetting correction and background subtraction was performed as described above. After subtraction of the corresponding blank field profile, only a contribution from the CXB remains (see Appendix A). Since the data are corrected for vignetting effects, the $\mathrm{CXB}$ surface brightness profile should be constant with radius. The profile was indeed found to be flat beyond $4^{\prime}$, where we can thus consider that the cluster emission is negligible. The residual background was thus estimated from the data within $4^{\prime}-7^{\prime}$ and subtracted from the profile. Starting from the central annulus, we re-binned the data in adjacent annulii so that i) at least a $S / N$ ratio of $3 \sigma$ is reached after background subtraction and ii) the width of the bin increases with radius, with $\Delta(\theta)>0.1 \theta$. Such a logarithmic radial binning insures a $S / N$ ratio in each bin roughly constant in the outer part of the profile, when the background can still be neglected.

The resulting surface brightness profile, $S(\theta)$, is shown in Fig. 2. The cluster emission is significantly detected up to $R_{\mathrm{det}}=3^{\prime}$ or $1.37 \mathrm{Mpc}$ for a critical density Universe. Beyond that radius it was not possible to create $S / N>3$ annulus of any width. The total count rate within the $R<3^{\prime}$ region and in the considered energy band is $0.30 \pm 0.006 \mathrm{ct} / \mathrm{s}$.

We fitted $S(\theta)$ with a $\beta$-model convolved with the XMM PSF, and binned as the observed profile. The PSF of each camera is modeled by a normalized King profile, with parameters depending on energy and off-axis angle (Ghizzardi 2001; Griffiths \& Saxton 2002). The overall PSF at each radius 


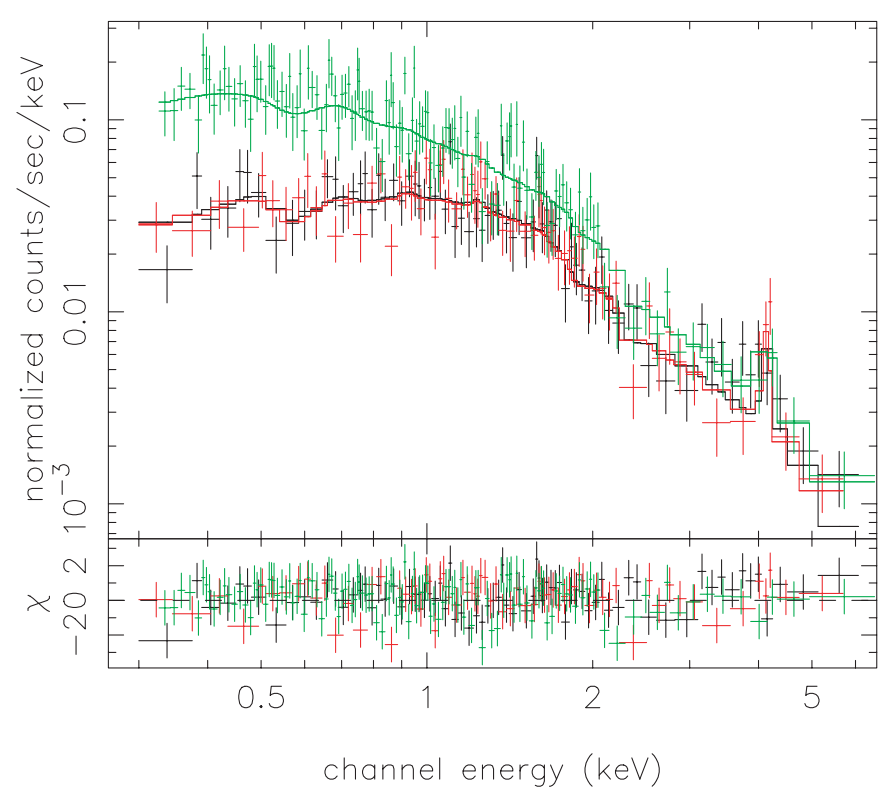

Fig. 3. XMM spectra of the cluster from the $\theta<2^{\prime}$ region. Black (red) [green] points: EPIC/MOS1(2)[pn] data. The EPIC spectra are background subtracted and corrected for vignetting as described in Sect. 2. Solid lines: best fit isothermal model with $k T=5.3 \mathrm{keV}$, an abundance of 0.47 times the solar value.

is obtained by summing the PSF of each camera, estimated at an energy of $1 \mathrm{keV}$, weighted by the respective cluster count rate in the [0.3-3] keV energy band. The on-axis overall PSF is plotted in Fig. 2 (dashed line). The convolution with the PSF takes into account the (small) PSF variation across the region considered. However, in practice only the MOS PSF variations are taken into account. We used the on-axis pn PSF, due to the lack of available parametrical fit of the off-axis data.

The fit of the cluster profile gives $\beta=0.78_{-0.04}^{+0.06}$ and a core radius of $\theta_{\mathrm{c}}=0.44_{-0.04^{\prime}}^{+0.06^{\prime}}$. The reduced $\chi^{2}$ is $\sim 1\left(\chi^{2}=20\right.$ for 21 d.o.f.). The convolved best fit model is plotted in Fig. 2 (solid line), together with the corresponding (unbinned and non-convolved) $\beta$-model (dotted line). As can be seen, the PSF mostly affects the core of the profile.

We then estimated the cluster surface brightness profile corrected for the effect of the PSF, that we will use in the following (Sect. 4). In principle, we should have deconvolved the observed profile. However since the cluster profile is well fitted by a $\beta$-model, we used a simpler procedure. For each radial bin, we estimated the ratio of the surface brightness corresponding to the non-convolved and convolved best fit $\beta$-model. The observed surface brightness profile was then corrected for the effect of the PSF, by simply multiplying the observed $S(\theta)$ value in each bin by the model ratio obtained for that bin.

\subsection{Mean temperature}

The overall MOS1, MOS2 and pn spectra, extracted from the event file, are shown in Fig. 3. The spectra are corrected for vignetting and background subtracted (see Sect. 2.3). To optimize the $S / N$ ratio, the integration region for the cluster was restricted to $2^{\prime}$ from the cluster center, and the residual $\mathrm{CXB}$

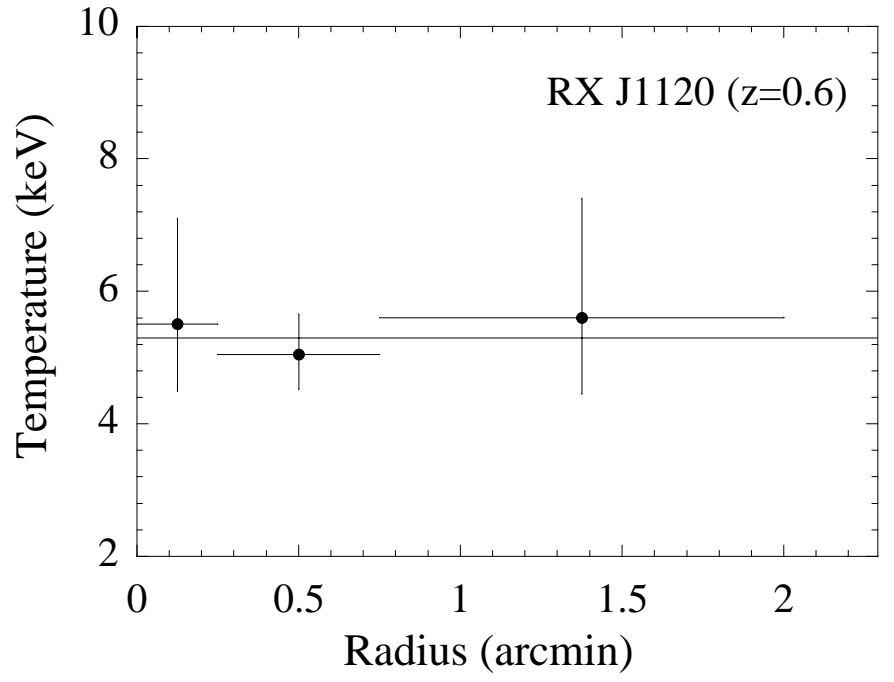

Fig. 4. Radial temperature profile as a function of angular radius, derived from XMM/EPIC data. The horizontal line corresponds to the mean value derived from fitting the overall spectrum of the region within $2^{\prime}$ in radius.

spectrum was estimated from the $4^{\prime}<\theta<11^{\prime}$ region. The spectra are binned so that the $S / N$ ratio is greater than $3 \sigma$ in each energy bin after background subtraction.

The spectra are jointly fitted with XSPEC using a redshifted MEKAL model (Mewe et al. 1985, 1986; Kaastra 1992; Liedahl et al. 1995). We let the relative normalization between the various instruments be free but assumed a common temperature and abundance. When letting the hydrogen column density $N_{\mathrm{H}}$ and redshift $z$ free, we obtained $N_{\mathrm{H}}=2.2 \pm 1.2 \times 10^{20} \mathrm{~cm}^{-2}$, in agreement with the $21 \mathrm{~cm}$ value $\left(N_{\mathrm{H}}=2.26 \times 10^{20} \mathrm{~cm}^{-2}\right.$ from Dickey \& Lockman 1990) and $z=0.61 \pm 0.03$, consistent with the optical value $(z=0.60)$. We then fixed these parameters to the $21 \mathrm{~cm}$ and optical values. The best fit gives $k T=5.3 \pm 0.5 \mathrm{keV}$ and an abundance of $0.47 \pm 0.19$. The reduced $\chi^{2}$ is $\sim 0.94$ ( $\chi^{2}=258$ for 276 d.o.f.).

The temperature values estimated separately from the MOS and pn spectra are consistent within the error bars $(k T=$ $5.8_{-0.7}^{+1.0} \mathrm{keV}$ for MOS data and $k T=4.5_{-0.5}^{+0.8} \mathrm{keV}$ for pn data). We note, however that a lower temperature is obtained with the pn data than with the MOS data. This is likely to be partly due to the remaining calibration systematic uncertainties in the XMM spectral responses. Indeed, it is known that the EPIC-pn and MOS cameras show a relative flux difference of $4 \%$ at low energies which increases with energy above $4.5 \mathrm{keV}$, resulting in a MOS spectral slope flatter than the pn (Saxton 2002; Griffiths et al. 2002).

\subsection{Temperature profile}

We then extracted the spectra in three concentric annuli, centered on the cluster $\mathrm{X}$-ray emission peak and fitted the data as described above. The corresponding temperature profile is shown in Fig. 4. It appears flat up to $2^{\prime}$, within the error bars.

The emission from the central annulus is affected by the PSF and may contaminate the other annulii, blurring out gradients if they exist. Moreover the energy dependence of 
the PSF, if not taken into account, might in principle bias the temperature estimate, since the photon redistribution is energy dependent. However, for the MOS1 instrument for instance, the Encircled Energy Fraction (EEF) within 15", the size of the first bin, is already about $70 \%$ (Ghizzardi 2001). The flux in the first bin is about $1 / 3$ of the second bin flux. We thus expect a contamination of only $\sim 10 \%$ in the second bin and we probably do not significantly underestimate possible gradients in the central part. Furthermore, the EEF varies only by $4.7 \%$ between $0.3 \mathrm{keV}$ and $5 \mathrm{keV}$, the minimum and maximum energy for the spectra. Neglecting the PSF energy dependence results in a negligible bias $(\sim 0.5 \%)$ at high energies. We emphasize that the XMM telescope PSF has a very weak energy dependence, so that indeed the correction is tiny and we are not facing the problem experienced in spectro-imagery with ASCA, for example.

\section{Scaling properties}

\subsection{The self-similar model}

The self-similar model is based on simple assumptions for cluster formation, derived from the top-hat spherical collapse model. The virialised part of a cluster, present at a given redshift, corresponds to a fixed density contrast as compared to the critical density of the Universe at that redshift and the internal shape of clusters of different masses and $z$ are similar. Self similarity applies to both the dark matter component and the hot intra-cluster medium, whose mass fraction is assumed to be constant. Consequently simple scaling laws relate each physical X-ray property, $Q$, to the cluster total mass (or temperature $T$ ) and redshift, in the form $Q \propto A(z) T^{\alpha}$.

This simple model thus makes a definitive prediction in terms of the evolution of cluster properties. First, the normalization (but not the slope) of the $Q-T$ scaling relations should evolve with $z$. In particular, for a critical density Universe, the virial radius at fixed $T$ should decrease with $z$ as $(z+1)^{-3 / 2}$, while the luminosity and central emission measure increase as $(z+1)^{3 / 2}$ and $(z+1)^{9 / 2}$ respectively. Furthermore once expressed in scaled coordinates ${ }^{3}$, the radial profile of any physical quantity (e.g density, temperature) should be the same at all redshifts.

In this section we will compare the structural and scaling properties of RX J1120.1+4318 to the properties of nearby clusters. We will consider the scaled emission measure and temperature profiles, the gas mass fraction and the $L_{X}-T$ relation. Results are given for both the EdS model and the $\Lambda \mathrm{CDM}$ model $^{4}$. The physical properties of RX J1120.1+4318 are summarized in Table 1.

\footnotetext{
3 The considered quantity is normalized according to the scaling relation estimated at the cluster temperature and redshift and the radius is expressed in units of the virial radius

${ }^{4}$ The comparison of distant and nearby cluster properties does not depend on the assumed $H_{0}$ value: scaled and physical quantities depend on $H_{0}$ via a multiplying factor, the same at all redshifts.
}

\subsection{The scaled emission measure profile}

Recently, Arnaud et al. (2002, hereafter AAN) studied the surface brightness profiles of a sample of 25 distant $(z=0.3-0.83)$ hot $(k T>3.5 \mathrm{keV})$ clusters observed with ROSAT, with published temperature from ASCA. They found that the scaled profiles of distant clusters are perfectly consistent with the average scaled profile of nearby clusters, for a flat low density Universe. For RX J1120.1+4318, we will make the same comparison, and we only briefly summarize the method.

\subsubsection{The cluster profile}

We used the surface brightness profile, corrected for the effect of the PSF (see Sect. 3.1). It is converted to emission measure $(E M)$ profile:

$$
E M(r) \propto S(\theta)(1+z)^{4} / \epsilon(T, z)
$$

where $\epsilon(T, z)$ is the emissivity in the energy band considered, taking into account the instrument response and interstellar absorption, and $r=\theta d_{\mathrm{A}}$, where $d_{\mathrm{A}}$ is the angular distance.

The $E M$ profile is then scaled according to the self-similar model. We use the standard scaling relations of cluster properties with redshift and temperature, with the empirical slope of the $M_{\mathrm{gas}}-T$ relation derived by Neumann \& Arnaud (2001). This empirical relation is consistent with the observed steepening of the $L_{X}-T$ relation and reduces significantly the scatter in the scaled profile of nearby clusters. The physical radius is thus scaled to the virial radius, $R_{\mathrm{V}}$, with $R_{\mathrm{V}} \propto \Delta_{z}^{1 / 2}(1+z)^{3 / 2} T^{1 / 2}$ and the emission measures by $\Delta_{z}^{3 / 2}(1+z)^{9 / 2} T^{1.38}$ (see AAN for details). This scaling depends on the density parameter $\Omega_{0}$ and the cosmological constant $\Lambda$, via the factor $\Delta_{z}=$ $\left(\Delta_{\mathrm{c}}\left(\Omega_{z}, \Lambda\right) \Omega_{0}\right) /\left(18 \pi^{2} \Omega_{\mathrm{z}}\right)$, where $\Delta_{\mathrm{c}}\left(\Omega_{z}, \Lambda\right)$ is the cluster density contrast at redshift $z$ and $\Omega_{z}$ the corresponding Universe density parameter. Analytical expression of $\Delta_{\mathrm{c}}\left(\Omega_{z}, \Lambda\right)$ can be found in Bryan \& Norman (1998). The scaled profiles also depend on these cosmological parameters via the angular distance used to convert angular radius to physical radius.

The scaled emission measure profiles of RX J1120.1+4318 derived for the EdS model and the $\Lambda \mathrm{CDM}$ model are displayed in the left and right panel of Fig. 5, respectively. The corresponding virial radii are given in Table 1 . As in AAN, the normalization of the $R_{\mathrm{V}}-T$ relation is taken from the simulation Evrard et al. (1996).

\subsubsection{Comparison with local data}

For each cosmological model, the scaled profile of RX J1120.1+4318 is compared in Fig. 5 with the corresponding average scaled profile of nearby clusters. This profile (full line), and the typical dispersion around it (dotted lines) is derived, as in AAN, from a sample of 15 hot nearby clusters observed with ROSAT (Neumann \& Arnaud 1999). The comparison is performed at radii beyond $\sim 0.1 R_{\mathrm{V}}$, where nearby clusters were found to obey self-similarity ${ }^{5}$.

\footnotetext{
5 A large dispersion was observed in the cluster core properties, which are dominated by non-gravitational physics.
} 

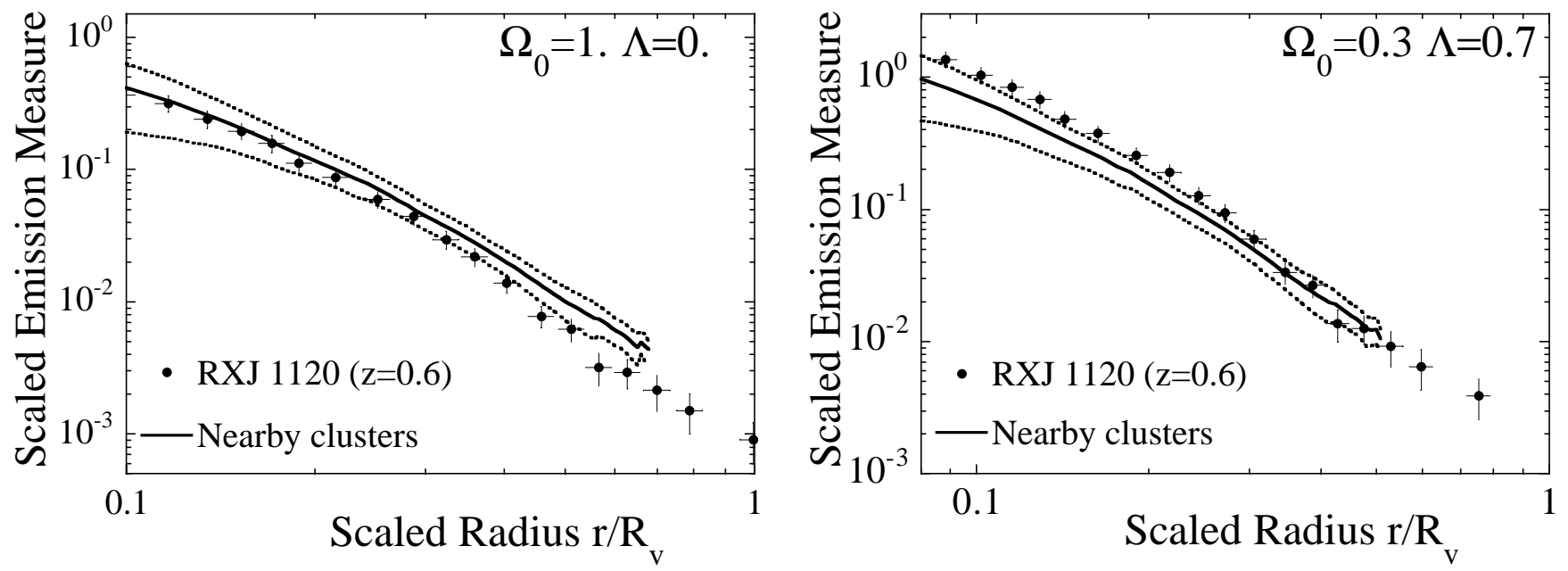

Fig. 5. Comparison between the scaled emission measure profile of RX J1120.1+4318 (data points) and the mean scaled profile of nearby hot clusters (full line, see text) for two cosmological models. The error bars on the scaled variables take into account the error on the temperature and on the surface brightness profile. The dotted lines correspond to the reference nearby profile, plus or minus the corresponding standard deviation. Left panel: results for a EdS Universe $\left(\Omega_{0}=1, \Lambda=0\right)$. Right panel: results for a $\Lambda$ CDM model $\left(\Omega_{0}=0.3, \Lambda=0.7\right)$.

To be meaningful, this comparison requires that the relative error in the calibration of XMM/EPIC and ROSAT/PSPC can be neglected. Our previous study of A1795 (Arnaud et al. 2001), where we performed a combined fit of the EPIC/MOS and ROSAT/PSPC spectra, indicates that the fluxes derived by the two instruments match at the $\sim \pm 5 \%$ level. Similar relative calibration uncertainties were derived by Snowden (2002).

For both cosmological models, the scaled profile of RX J1120.1+4318 is consistent with the local reference profile, taking into account its intrinsic scatter. The RX J1120.1+4318 data thus reinforce the validity of the self-similar model. However, some significant discrepancy in shape can be noted. At large radii the cluster profile falls off more rapidly than the reference profile. This corresponds to its higher $\beta$ value, $\beta=0.78$, as compared to the universal value of $\beta=2 / 3$, which fits well the reference curve (Neumann \& Arnaud 1999). The scaled core radius, $x_{\mathrm{c}}=r_{\mathrm{c}} / R_{\mathrm{V}}$, on the other hand, is similar to the value $\left(x_{\mathrm{c}}=0.12\right)$ of the reference profile: we obtain $x_{\mathrm{c}} \sim 0.11$ (EdS model) and $x_{\mathrm{c}} \sim 0.14$ ( $\Lambda \mathrm{CDM}$ model). This might simply be due to intrinsic scatter in the properties of distant clusters. A typical scatter of $\sim 20 \%$ is indeed observed in the slopes of nearby cluster profiles (Neumann \& Arnaud 1999). A scatter in cluster properties is observed as well in numerical simulations of cluster formation (Navarro et al. 1997). However, this discrepancy could also indicate a systematic departures from the self-similar model considered. With data on a single cluster, we obviously cannot distinguish between these two possibilities.

It is also interesting to compare in more detail the results obtained for the two cosmological models. The relative position of the scaled profile of RX J1120.1+4318 with respect to the reference profile is higher (in $y$ axis) for the $\Lambda \mathrm{CDM}$ model than for the EdS model. As discussed by AAN, the derived scaled profiles of distant clusters depend on the cosmological parameters, mostly via the angular distance $d_{\mathrm{A}}$. The typical dependence is $\propto d_{\mathrm{A}}^{3}$ at fixed scaled radius. At $z=0.6$, this $d_{\mathrm{A}}^{3}$ factor is about $85 \%$ higher for a $\Lambda \mathrm{CDM}$ model than for an
EdS model. The $d_{\mathrm{A}}^{3}$ dependence is strictly exact for a profile of logarithmic slope -3 (e.g. in the cluster external region for a $\beta$-model with $\beta=2 / 3$ ). Due to the steepest slope of the RX J1120.1+4318 profile at large radii, the effect of varying the cosmological parameters is slightly smaller, of the order of $\sim 60 \%$ (Fig. 5). The effect is smaller than the intrinsic dispersion in the nearby profiles at radius below typically $0.2 R_{\mathrm{V}}$, and becomes only marginally larger than the $\sim \pm 25 \%$ dispersion at higher radii. As emphasised by AAN, no definitive conclusion on the cosmological parameters can then be drawn from the observation of a single distant cluster. We simply note that the profile of RX J1120.1+4318 is in better agreement with the local reference profile at large radii for a $\Lambda \mathrm{CDM}$ model than for an EdS model. In the latter model, the profile appears to deviate more and more from the reference profile above $\sim 0.4 R_{\mathrm{V}}$. The better agreement observed for the $\Lambda \mathrm{CDM}$ model can simply be an artifact, due to the intrinsic dispersion in the cluster properties, or again due to systematic departure from the self-similar model. However, it is consistent with the statistical analysis of the scaled profiles of distant clusters, performed by AAN, which clearly favors a low density Universe.

Finally, we note the statistical quality of the scaled emission measure profile, as compared to ROSAT/ASCA data. The profile of RX J1120.1+4318 is traced nearly up to the virial radius, further out than the mean profile of nearby clusters observed with ROSAT and the errors bars on most of the data points are smaller than the typical dispersion in the reference profile. Note also the very good sampling of the profile. Using the same criteria (at least $3 \sigma$ detection in each bin), most of the ROSAT profiles of distant clusters (AAN, Fig. 4) are much more sparsely sampled.

\subsection{The scaled temperature profile}

Cluster temperature profiles can now be measured with high precision with XMM. First observations of nearby clusters 


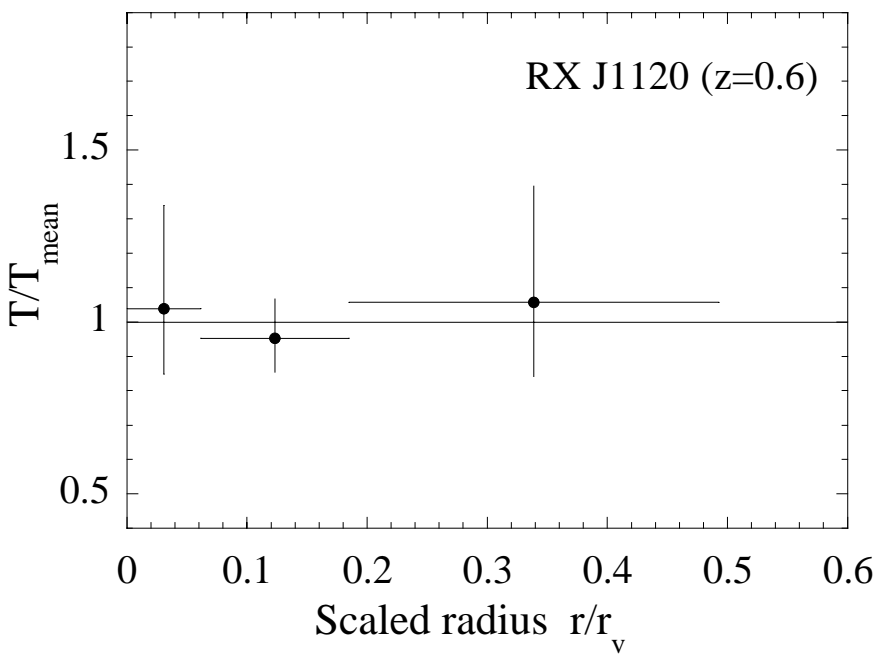

Fig. 6. Scaled temperature profile. The temperature is normalized to the mean value and the radius is expressed in units of the virial radius ( $\Lambda$ CDM model, see Table1).

indicate that the profiles are isothermal (within typically 10\%) up to half the virial radius (Arnaud et al. 2001; Arnaud 2001).

With XMM we can now also study the evolution of the temperature profiles. The cluster temperature profile, normalized to the mean temperature, is plotted in Fig. 6 as a function of scaled radius ( $\Lambda \mathrm{CDM}$ model). The temperature profile of $\mathrm{RX} \mathrm{J1120} \mathrm{is}$ mapped up to about $0.5 R_{\mathrm{V}}$. This is the first time that a temperature profile can be measured at such high redshift. Although the errors bars are large, this distant cluster appears isothermal, as observed for nearby clusters. This is consistent with the self-similarity in shape expected in the simple model of cluster formation.

\subsection{The gas mass fraction}

\subsubsection{VT method}

We first estimated the virial radius $\left(R_{\mathrm{V}}\right)$ and mass $\left(M_{\mathrm{V}}\right)$ from the best fit temperature and the theoretical $R_{\mathrm{V}}-T$ and $M_{\mathrm{V}}-T$ scaling relations at the cluster redshift, as described in Sect. 4.1. We recall that these relations correspond to a fixed density contrast at redshift $z$ and are derived using the virial theorem (VT):

$$
\frac{G \mu m_{\mathrm{p}} M_{\mathrm{V}}}{2 R_{\mathrm{V}}}=\beta_{\mathrm{T}} k T
$$

where the normalization factor is assumed to be $\beta_{\mathrm{T}}=1.05$, from the simulation of Evrard et al. (1996). In that case:

$M_{\mathrm{V}}=2.98 \times 10^{15} \Delta_{z}^{-1 / 2}(1+z)^{-3 / 2}\left(\frac{k T}{10 \mathrm{keV}}\right)^{3 / 2} M_{\odot}$.

The best fit temperature corresponds to the emission weighted temperature within $2^{\prime}$, which is about half of the virial radius (see Table 1). Since $\sim 90 \%$ of the emission lies within that radius, this temperature is a good estimate of the overall emission weighted value. Within that radius, there is no significant evidence of strong gradients. Nevertheless, if the temperature profile decreases beyond $2^{\prime} \sim 0.5 R_{\mathrm{V}}$, the measured temperature would be an overestimate of the mass weighted temperature (considered in the simulations). However, if the shape of the temperature profile does not evolve with $z$, the corresponding bias in the VT mass estimate would be the same at all redshifts and will not affect the following comparison with nearby cluster properties.

The central hydrogen density, $n_{\mathrm{H}, 0}{ }^{6}$ and the corresponding gas mass within the virial radius, $M_{\text {gas }}$, are derived from the best fit $\beta$-model parameters (central surface brightness, $\beta$ and core radius), assuming a cluster extent equal to the virial radius. The errors on these quantities, due to that on the surface brightness profile, are derived as in Elbaz et al. (1995). The error due to the uncertainty on the temperature, which appears in the emissivity factor, is negligible. We emphasize that the estimate of the gas mass does not require severe extrapolation of the data and is therefore robust. The cluster emission is detected virtually up to the virial radius for an EdS model $\left(R_{\text {det }} \sim R_{\mathrm{V}}\right)$ and up to about $0.75 R_{\mathrm{V}}$ for a $\Lambda \mathrm{CDM}$ model (Table 1 ). The gas mass within that radius is already $70 \%$ of the gas mass within $R_{\mathrm{V}}$.

The central hydrogen density, gas mass, virial radius, virial mass and gas mass fraction, $f_{\text {gas }}=M_{\text {gas }} / M_{\mathrm{V}}$, are given in Table 1 for the EdS and $\Lambda$ CDM models. The uncertainty on this last quantity is dominated by the uncertainty on the temperature, through the estimate of the virial mass.

\subsubsection{BM method}

We also estimated the total mass using the hydrostatic equilibrium (HE) equation and the isothermal $\beta$-model (BM method):

$$
\frac{G \mu m_{\mathrm{p}} M_{\mathrm{V}}}{2 R_{\mathrm{V}}}=\frac{3 \beta}{2} \frac{\left(R_{\mathrm{V}} / r_{\mathrm{c}}\right)^{2}}{1+\left(R_{\mathrm{V}} / r_{\mathrm{c}}\right)^{2}} k T
$$

where $\beta$ and $r_{\mathrm{c}}$ are the slope and core radius parameters of the gas distribution (derived Sect. 3.1).

The corresponding virial mass and radius, corresponding to the same density contrast as in the VT approach, are given in Table 1. Comparing Eqs. (2) and (4) and neglecting the terms $\left(r_{\mathrm{c}} / R_{\mathrm{V}}\right)^{2}$, the ratio of the virial masses (or radius) derived from the VT and BM method is:

$$
\frac{R_{\mathrm{V}}(B M)}{R_{\mathrm{V}}(V T)} \sim\left(\frac{3 \beta}{2 \beta_{\mathrm{T}}}\right)^{1 / 2} ; \quad \frac{M_{\mathrm{V}}(B M)}{M_{\mathrm{V}}(V T)} \sim\left(\frac{3 \beta}{2 \beta_{\mathrm{T}}}\right)^{3 / 2}
$$

The VT and BM methods give very similar results (see Table 1). As compared to the VT estimate, the virial radius and mass, estimated using the BM method, are $\sim 5 \%$ and $\sim 15 \%$ higher, respectively. The central hydrogen density is unchanged. The gas mass within the virial radius is about $\sim 5 \%$ higher (slightly larger integration region) and the gas mass fraction is decreased by about $10 \%$.

\subsubsection{Comparison with the local gas mass fraction}

The gas mass fraction of RX J1120.1+4318 can be compared to the $90 \%$ confidence region derived by Arnaud \& Evrard (1999, hereafter AE) for hot $(k T>4 \mathrm{keV})$ nearby clusters

\footnotetext{
$6 n_{\mathrm{H}, 0}=0.85 n_{\mathrm{e}, 0}$, where $n_{\mathrm{e}, 0}$ is the central electronic density for an ionised plasma with the observed abundance.
} 
Table 1. Summary of the physical properties of RX J1120.1+4318 for an $\operatorname{EdS}$ model $\left(\Omega_{0}=1\right)$ and a $\Lambda$ CDM model $\left(\Omega_{0}=0.3, \Lambda=0.7\right)$.

\begin{tabular}{lll}
\hline \hline Model & EdS & $\Lambda$ CDM \\
\hline$r_{\mathrm{c}}(\mathrm{kpc})$ & $203_{-21}^{+24}$ & $250_{-26}^{+30}$ \\
$R_{\mathrm{det}}(\mathrm{Mpc})$ & 1.37 & 1.68 \\
$n_{\mathrm{H}, 0}\left(10^{-3} \mathrm{~cm}^{-3}\right)$ & $6.9_{-0.5}^{+0.6}$ & $6.2_{-0.4}^{+0.5}$ \\
\hline & \multicolumn{2}{c}{ Virial Theorem } \\
\cline { 2 - 3 }$R_{\mathrm{V}}(\mathrm{Mpc})$ & $1.40 \pm 0.07$ & $2.28 \pm 0.11$ \\
$M_{\mathrm{V}}\left(10^{14} M_{\odot}\right)$ & $5.8 \pm 0.8$ & $9.5 \pm 1.3$ \\
$M_{\text {gas }}\left(<R_{\mathrm{V}}\right)\left(10^{14} M_{\odot}\right)$ & $0.85 \pm 0.04$ & $1.85 \pm 0.12$ \\
$f_{\text {gas }}(\%)$ & $14.6 \pm 2.1$ & $19.6 \pm 3.0$ \\
& $\mathrm{HE}+$ isothermal $\beta-$ model \\
\cline { 2 - 3 }$R_{\mathrm{V}}(\mathrm{Mpc})$ & $1.47 \pm 0.08$ & $2.40 \pm 0.12$ \\
$M_{\mathrm{V}}\left(10^{14} M_{\odot}\right)$ & $6.1 \pm 0.9$ & $11.0 \pm 1.7$ \\
$M_{\text {gas }}\left(<R_{\mathrm{V}}\right)\left(10^{14} M_{\odot}\right)$ & $0.89 \pm 0.04$ & $1.94 \pm 0.12$ \\
$f_{\text {gas }}(\%)$ & $13.3 \pm 2.1$ & $17.6 \pm 3.0$ \\
\hline$L_{\mathrm{X}}\left(10^{45} \mathrm{ergs} / \mathrm{s}\right)$ & $1.39 \pm 0.08$ & $2.14 \pm 0.12$ \\
$C$ & $1.7_{-0.4}^{+0.6}$ & $2.4_{-0.5}^{+0.8}$ \\
$C_{\text {mod }}(z)$ & $2.0 \pm 0.3$ & $1.65 \pm 0.25$ \\
\hline
\end{tabular}

using the same total mass estimate methods. AE found ${ }^{7}$ : $f_{\text {gas }}=20.1[-2.5,+3.0] \%$ using the VT method and $f_{\text {gas }}=$ $21.5[-3.5,+4.5] \%$ with the BM method. For both the VT and $\mathrm{BM}$ methods, the value derived for RX J1120.1+4318 is perfectly consistent with the AE local value for a $\Lambda$ CDM model, but is significantly lower for an EdS model.

However, the temperature of RX J1120.1+4318 ( 5.3 keV) is lower than the median temperature of the $\mathrm{AE}$ hot cluster sample $(\sim 8 \mathrm{keV})$. We are thus not exactly comparing clusters of same temperature and there is some indication that the gas mass fraction varies with $T$. The empirical slope of the $M_{\text {gas }}-T$ relation derived by Neumann \& Arnaud (2001) corresponds to $f_{\text {gas }} \propto T^{0.44}$, if the classical $M_{\mathrm{V}}-T$ relation is not modified. This variation is consistent with the variation, $f_{\text {gas }} \propto T^{0.41 \pm 0.16}$, found by Mohr et al. (1999) using the virial mass estimate. A variation of $f_{\text {gas }}$ with $T$ is also readily apparent in Fig. 3 of AE. Correcting the AE $f_{\text {gas }}$ values for a possible bias of $\sim(5.3 / 8)^{0.44}$, gives a local value of $f_{\text {gas }}=16.8 \%(\mathrm{VT}$ approach), now in agreement with the RX J1120.1+4318 estimate for both a $\Lambda$ CDM model and an EdS model, although marginally in the latter case.

The gas mass fraction has been proposed from some time (Pen 1997; Rines et al. 1999) as a novel distance indicator. The method is based on the assumed constancy of this quantity with $z$, while its estimate from $\mathrm{X}$-ray data depends on the assumed angular distance. As Mohr et al. (2000) pointed out, the region considered to compare $f_{\text {gas }}$ at different redshifts must be fixed in terms of scaled radius, with the evolution of the

\footnotetext{
${ }^{7}$ These values were derived for an EdS model, but the local value is not sensitive to the cosmological parameters. The definition of the virial region is also slightly different but close enough to have no impact on our conclusion: our definition corresponds to a density contrast of 178 at $z=0$ for an EdS model, while the value quoted are estimated at a density contrast of 200 .
}

virial radius properly taken into account, to avoid biases due to the variation of the gas mass fraction with physical radius. The comparison performed above, where the gas mass fractions are estimated within the virial radius, does not suffer from this bias $^{8}$. However, as illustrated above, the comparison is further complicated by possible variation of $f_{\text {gas }}$ with cluster temperature. A much better understanding of the $f_{\text {gas }}-T$ relation, both in the local and distant Universe is required, before any conclusion can be drawn on the cosmological parameters.

\subsection{The $L_{x}-T$ relation}

\subsubsection{Cluster luminosity}

We computed the bolometric luminosity, $L_{X}$, within the virial radius (given Table 1). The observed count rate in the [0.3-3] keV band, obtained by integrating the surface brightness profile up to the detection radius, is converted to bolometric luminosity using the best fit MEKAL spectral model and the instrument response. The contribution beyond the detection region is estimated from the best fit $\beta$-model, but is totally negligible $\left(0.7 \%\right.$ for the $\Lambda \mathrm{CDM}$ model). The error on $L_{X}$ includes both the statistical error on the count rate and on the temperature.

\subsubsection{Evolution of the $L_{x}-T$ relation}

As in AAN and in Sadat et al. (1998), we divided this luminosity by the luminosity estimated from the local $L_{X}-T$ relation of AE and the cluster temperature: $L_{\mathrm{X}}=1.15 \times 10^{45}(T / 6 \mathrm{keV})^{2.88}$. The resulting factor $C$ is given in Table 1 for the two cosmological models considered. The contribution to the error due to the uncertainties on $T$ and $L_{\mathrm{X}}$ are summed quadratically in the $\log$ space.

This $C$ factor can be compared to the evolution of the normalization of the $L_{X}-T$ relation, $C_{\bmod }(z)=\Delta_{z}^{1 / 2}(1+z)^{3 / 2}$, expected in the self-similar model (AAN). This theoretical value, estimated at the cluster redshift, is given in Table 1 for the EdS and $\Lambda C D M$ models. The "error" bars corresponds to plus or minus the intrinsic scatter in $L_{X}$ estimated by AE. The luminosity of RX J1120.1+4318 is in good agreement with the expected evolution for an EdS Universe, and in the upper range of the expected evolution for a $\Lambda$ CDM Universe. For an EdS model, the luminosity is also marginally consistent with no evolution.

\subsubsection{Comparison with recent works}

Several groups quantified the evolution of the $L_{X}-T$ relation using ROSAT/ASCA data (Sadat et al. 1998; Reichart et al. 1999; Fairley et al. 2000; AAN). There is a general consensus that no significant evolution is observed for an EdS model. However, as emphasized by AAN, both the luminosity estimate and the theoretical evolution depend on the assumed cosmology and a different conclusion is reached for a $\Lambda \mathrm{CDM}$ model.

\footnotetext{
${ }^{8}$ Note that the dependence of $f_{\text {gas }}$ on $\Omega_{0}$ and $\Lambda$ in the present approach (given in Neumann \& Arnaud 2000) is indeed different than the $d_{\mathrm{A}}^{3 / 2}$ dependence derived by Pen (1997) and Rines et al. (1999).
} 
In that case, AAN found a significant evolution, consistent with the self-similar model. Our results agree with this finding. They are also consistent with the results of Reichart et al. (1999): they found an evolution of $C=(1+z)^{\left(0.91-1.12 q_{0}\right)_{-1.22}^{+0.54}}$ in the redshift range $z<0.5$ or $C=2.0_{-0.9}^{+0.6}$ extrapolated at $z=0.6$ for the $\Lambda \mathrm{CDM}$ model considered here. This is similar to the value, $C=2.4_{-0.5}^{+0.8}$, derived for RX J1120.1+4318.

On the other hand, Borgani et al. (2001b), using recent CHANDRA data up to $z=1.26$, did not find any evidence of significant evolution, for the EdS model but also for the $\Lambda$ CDM model. We note first the large uncertainties on the CHANDRA data, especially above $z=1$. Moreover, there is a large dispersion, with data points above and below the local $L_{X}-T$ curve for the $\Lambda \mathrm{CDM}$ model. To illustrate this point, let us consider the two clusters RX J0848+4456 $(z=0.57, k T=3.6 \pm 0.5 \mathrm{keV})$ and MS1137.5+6625 $(z=$ $\left.0.78, k T=5.7_{-0.6}^{+0.8} \mathrm{keV}\right)$. They are both relaxed clusters, with precise $k T$ measurements, and are in the same redshift and temperature range than RX J1120.1+4318. The $C$ value of MS1137.5+6625, $C=2.0_{-0.8}^{+0.7}$, is perfectly consistent with the expected evolution: $C_{\bmod }(z)=1.88$ at the cluster redshift and is similar to the value obtained for RX J1120.1+4318. On the other hand, the luminosity of RX J0848+4456 is particularly low for its measured temperature: $C=0.6_{-0.2}^{+0.4}$, corresponding to a negative evolution and of course inconsistent with the expected positive evolution of $C_{\bmod }(z)=1.6$. The measured evolution is sensitive to systematic uncertainties on the temperature $\left(C \propto T^{-3}\right)$. RX J0848+4456 was observed with ACIS-I (Holden et al. 2001), for which there are still large calibration uncertainties, especially at low energies. This is a potential worry for temperature measurements of high $z$ clusters with this instrument. The temperature estimate of MS1137.5+6625, observed with ACIS-S (Borgani et al. 2001b), is a priori more secure. MS1137.5+6625 was also in the AAN sample and the Chandra/ACIS-S data are consistent with the ASCA data. First cross calibration studies of ACIS-S and XMM/EPIC also show a good agreement between these instruments (Snowden 2002). Further cross-calibration studies and accurate measurements on a larger cluster sample are definitively required to assess if the presently observed dispersion is real.

\subsection{Consistency of the results}

In our comparisons with the nearby cluster properties, we found that the scaled $E M$ profile of RX J1120.1+4318, as well as its gas mass fraction and luminosity, are consistent with the predictions of the self-similar model of cluster formation. Since all three observables are not independent - they are directly related to the gas density - the consistency of our results is a priori not surprising. It is, however not entirely trivial, as we discuss now.

A good match of the scaled EM profile of RX J1120.1+4318 with the local profile, would imply that i) the shape of the gas profile is similar to that of nearby clusters ii) the $E M$ and thus gas density scales with $z$ as expected. Therefore its luminosity (integrated emission measure profile) and gas mass (integrated density profile) would naturally be found to follow the standard evolution. Since the standard evolution of the gas mass is the same than the evolution assumed to compute the virial mass, we would also find a gas mass fraction consistent with the local value. However, the emission measure, luminosity and possibly gas mass fraction also depend on the temperature. We emphasize that independent studies of the evolution of these quantities are thus expected to yield consistent results, but only if one considers a consistent scaling with $T$ for the three quantities. The present analysis is consistent in that sense. As mentioned above, the theoretical model used to scale the $E M$ profiles $\left(M_{\mathrm{gas}} \propto T^{1.94}\right)$ is consistent with the slope of the local $L_{\mathrm{X}}-T$ relation (used to normalize the cluster luminosity) and corresponds to $f_{\text {gas }} \propto T^{0.44}$ (used to estimate the local gas mass fraction at the cluster temperature).

In spite of the general agreement with the self-similar model, some differences appear when comparing the results of the EdS and $\Lambda$ CDM models. The analysis of the gas mass fraction and scaled profiles would rather favor a $\Lambda$ CDM model. However for such a model, the luminosity is in the upper range of the expected evolution and a better agreement is observed for an EdS model. This is actually a direct consequence of the specific shape of RX J1120.1+4318. The concentration of the gas distribution of RX J1120.1+4318 is slightly larger than the reference profile, yielding a higher luminosity as compared to the gas mass. These differences can also be understood by looking at the scaled profiles. As outlined above, the scaled profile of RX J1120.1+4318 is in a good agreement with the nearby profile at high radii for a $\Lambda$ CDM model. For an EdS model, the scaled profile lies more and more below the reference curve at $r \gtrsim 0.4 R_{\mathrm{V}}$, indicating a lower gas density than expected. Since the external regions contribute most to the gas mass ( $\sim 75 \%$ of the mass lies beyond $\left.0.3 R_{\mathrm{V}}\right)$, this explains why the gas mass fraction for an EdS model is marginally too low, even as compared to the corrected $\mathrm{AE}$ local value, while the agreement is better for a $\Lambda \mathrm{CDM}$ model. On the other hand, for an EdS model, the cluster scaled profile matches better the local profile in the central region (see Fig. 5), which contributes most to the X-ray luminosity.

\section{Core properties and non-gravitational effects}

\subsection{Radiative cooling}

The XMM data suggest that RX J1120.1+4318 does not host a cooling flow. There is indeed no indication of a temperature drop in the central bin and a good fit is obtained with a $\beta$-model, down to the center, with no indication of central excess.

We computed the central cooling time, $t_{\text {cool }}$ corresponding to the measured central density: $t_{\text {cool }}=2.9 \times 10^{10} \mathrm{yrs} \sqrt{T} / n_{\mathrm{H}}$, where $T$ is in $\mathrm{keV}$ and $n_{\mathrm{H}}$ in $\mathrm{cm}^{-3}$ (Sarazin 1986). We obtained $t_{\text {cool }} \sim 1 \times 10^{10} \mathrm{yrs}$. The age of the Universe at the cluster redshift is $t=6.5 \times 10^{9}$ yrs for the EdS model and $t=1.1 \times$ $10^{10} \mathrm{yrs}$ for the $\Lambda \mathrm{CDM}$ model. The cooling time, even at the cluster center where it is close to its minimum value, is thus similar to or larger than the age of the Universe. We thus do 


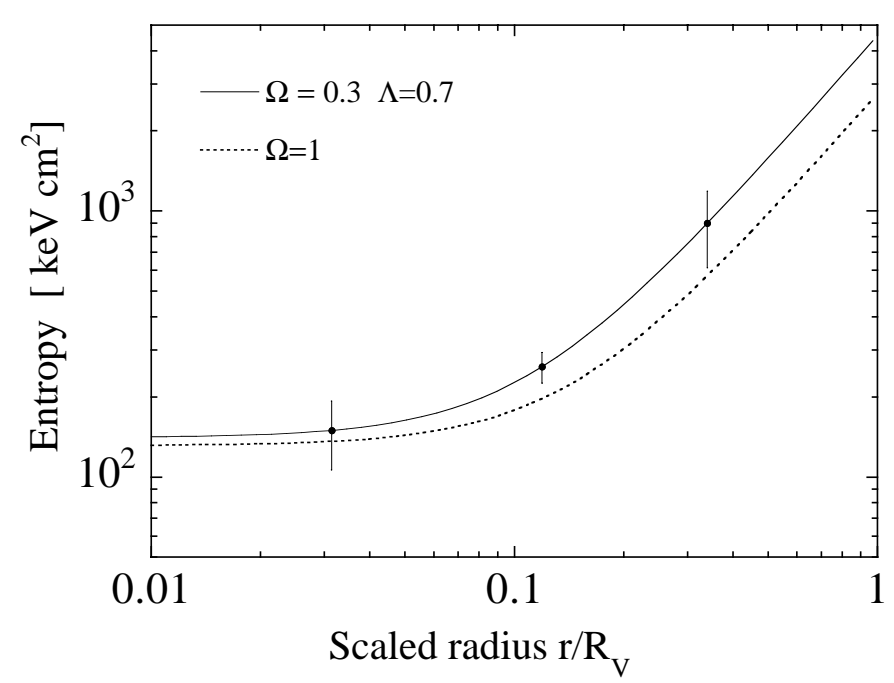

Fig. 7. Entropy profile of RX J1120.1+4318, derived from the best fit $\beta$-model and assuming isothermality. The radius is scaled to the virial radius for the EdS (dotted line) and $\Lambda \mathrm{CDM}$ (full line) models. The error bars corresponds to the error bars on the temperature profile, plotted at the central radii of the bins considered.

not expect a strong cooling flow in the center, in spite of the apparent relaxed state of the cluster.

\subsection{Entropy and non-gravitational heating}

As mentioned in the introduction, the simplest self-similar model of cluster formation fails to explain all the observed properties of the nearby cluster population. A definitive evidence of breaking of self-similarity is the entropy excess (the "entropy floor") detected in the core of cool systems with a baseline entropy of about $70-140 \mathrm{keV} \mathrm{cm}{ }^{2}$ (Ponman et al. 1999; Lloyd-Davies et al. 2000). The origin of this break of similarity is not yet understood.

With the present data, we can for the first time study the entropy profile of a $z=0.6$ cluster, ie look at cluster entropy evolution. Note that RX J1120.1+4318 appears to be in a relaxed state and the entropy distribution is not likely to be affected by shocks induced during recent mergers. The cluster entropy profile, as a function of scaled radius, is plotted in Fig. 7 for the EdS and $\Lambda$ CDM cosmologies. The entropy $S=T / n_{\mathrm{e}}^{2 / 3}$ is estimated from the best fit $\beta$-model and the cluster mean temperature. The uncertainty on the profile is dominated by the uncertainty on the temperature distribution. Typical errors, corresponding to the temperature error in each bin of the temperature profile are indicated in the figure.

One observes a nearly constant entropy level of about $140 \mathrm{keV} \mathrm{cm}{ }^{2}$ within the central $r<0.1 R_{\mathrm{V}}$ region and a rising entropy profile beyond that region. This shape is not surprising, the entropy profile shape reflects the shape of the inverse of the density profile, which has a core radius of about $0.1 R_{\mathrm{V}}$. The core entropy is, however, in remarkable agreement with the entropy "floor" measured in nearby clusters. The same agreement was noted by Arabadjis et al. (2002) in the case of EMSS $1358+6245$ at $z=0.33$.
If confirmed on more clusters, this coincidence has potentially substantial implications for the physics of cluster formation. We first note that it is consistent with the expectation of the early pre-heating scenario, where the gas is pre-heated at a given entropy level before collapse (e.g. Borgani et al. 2001a; Tozzi \& Norman 2001). At the beginning of the cluster formation, the entropy floor prevents shock heating and the collapse is adiabatic. The initial entropy is thus preserved in the core. When the mass of the system increases, the infall of incoming shells becomes supersonic and a shock regime begins (gravitational heating). The external profile follows the classical "self-similar" rising profile, as we observe. The core entropy of RX J1120.1+4318 suggests that the entropy floor was already established at high $z$ : at least $z \gtrsim 0.6$ and probably much before, since in a hierarchical scenario the core of the cluster must have collapsed earlier. Note also that the metal abundance found for this distant cluster is similar to the abundance observed in nearby clusters (e.g. de Grandi \& Molendi 2001 and reference therein). If early galactic winds are responsible for the gas pre-heating (e.g. Kaiser 1991; Evrard \& Henry 1991), we do expect that this is accompanied by an early enrichment. Finally, our data suggest that pre-heating in hot clusters has mostly an effect on the core properties up to high $z$. This might explain why AAN verified self-similarity of the $E M$ profile of hot clusters above $0.1 R_{\mathrm{V}}$ up to $z=0.8$.

Radiative cooling has been also proposed to explain the entropy "floor": it can remove low entropy gas from near the cluster center, triggering the inflow of higher entropy material (Pearce et al. 2000). RX J1120.1+4318, contrary to most nearby relaxed clusters (and EMSS 1358+6245), is a cluster, for which radiative cooling has probably not yet affected the core properties (see above). This suggests that radiative cooling cannot be the dominant process in the establishment of the entropy floor.

\section{Conclusion}

XMM-Newton data allow us to measure, with unprecedented accuracy at a redshift of $z=0.6$, the gas and temperature distribution of the distant cluster RX J1120.1+4318. The cluster has a regular spherical morphology, suggesting it is in a relaxed state. The cluster temperature is $k T=5.3 \pm 0.5 \mathrm{keV}$ and there is no significant evidence of temperature radial gradient up to half the virial radius. The surface brightness profile, measured nearly up to the virial radius, is well fitted by a $\beta$-model with $\beta=0.78_{-0.04}^{+0.06}$.

The RX J1120.1+4318 data reinforce the validity of the self-similar cluster formation model. For both an EdS and a $\Lambda \mathrm{CDM}$ model, the scaled emission measure profile beyond $\sim 0.1 R_{\mathrm{V}}$ is consistent with the nearby cluster reference profile, taking into account its intrinsic scatter. Consistently, the gas mass fraction is in agreement with the local value (although marginally for an EdS Universe), and the luminosity of the cluster, taking into account its temperature, is consistent with the expected evolution of the $L_{\mathrm{X}}-T$ relation.

There is no evidence of a cooling flow at the cluster center, in spite of its apparent relaxed state. This is consistent with the estimated cooling time, larger than the age of the Universe 
at the cluster redshift, indicating that radiative cooling has not yet affected the cluster properties. The entropy profile shows a flat core with a central entropy of $\sim 140 \mathrm{keV} \mathrm{cm}{ }^{2}$, remarkably similar to the entropy floor observed in nearby clusters. This favors early pre-heating models for the establishment of this entropy floor and suggests that pre-heating in hot clusters has mostly an effect on the core properties up to high $z$.

A statistical study of large sample, as the SHARC cluster sample, covering a wide range of redshift and luminosity (thus temperature) is required to better constrain the physics of cluster formation, from the evolution of cluster properties. However, the present data already demonstrate that XMM provides the statistical quality required by such study.

Acknowledgements. The present work is based on observations obtained with XMM-Newton, an ESA science mission with instruments and contributions directly funded by ESA Member States and the USA (NASA). We acknowledge financial support from the "Programme National de Cosmologie" funded by INSU, CEA and CNES. AKR acknowledges partial financial support from the NASA XMM-Newton GO program. DJB acknowledges the support for NASA contract NAS8-39073.

\section{Appendix A: Background subtraction method}

In this section we detail the background subtraction method. This method applies after screening for flares. We suppose we have a "template" event file, for the background estimate, obtained by collecting several high latitude observations. In the following, the data are supposed to be corrected for vignetting effect, as described in Arnaud et al. (2001) and in Sect. 2.2: each event detected with energy $E$ at location $x, y$ is weighted by a coefficient $w(x, y, E)$ which is the ratio of the effective area at position $(x, y)$ to the central effective area, for the energy $E$. The Cosmic X-ray Background (CXB) varies across the sky, but can be considered as uniform at the scale of $30^{\prime}$, the size of the field of view. The non X-ray background (NXB) is not uniform in the FOV, but is not vignetted.

For the template file, the corrected count rate, measured at a given location $(x, y)$ and energy $E, T(x, y, E)$, is the sum of the CXB and NXB contributions:

$T(x, y, E)=T_{\mathrm{CXB}}(x, y, E)+T_{\mathrm{NXB}}(x, y, E) w(x, y, E)$.

Since the data are corrected for vignetting effects, the CXB component is, apart from statistical fluctuations, uniform over the FOV and corresponds to the average CXB for the blank field observations:

$T_{\mathrm{CXB}}(x, y, E) \equiv T_{\mathrm{CXB}}(E)$.

The NXB component is the NXB for the observations, $T_{\mathrm{NXB}}(x, y, E)$, multiplied by the weight factor.

Similarly for the observation data set, the count rate is:

$$
\begin{aligned}
O(x, y, E)= & S(x, y, E)+O_{\mathrm{CXB}}(x, y, E) \\
& +O_{\mathrm{NXB}}(x, y, E) w(x, y, E)
\end{aligned}
$$

with

$O_{\mathrm{CXB}}(x, y, E) \equiv O_{\mathrm{CXB}}(E)$ where $S(x, y, E)$ is the source contribution and $O_{\mathrm{CXB}}(E)$ corresponds to the CXB at the pointing position of the considered observation. Since the $\mathrm{CXB}$ varies across the sky, a priori $O_{\mathrm{CXB}}(E) \neq T_{\mathrm{CXB}}(E)$. The quiescent NXB can be reasonably considered to have the same spectral and spatial characteristics for all observations but there is evidence of long term variations (D.Lumb 2002). We can thus write:

$O_{\mathrm{NXB}}(x, y, E) \equiv Q T_{\mathrm{NXB}}(x, y, E)$.

The normalization factor, $Q$, is estimated by considering the total count rate in the whole FOV in the high energy band, where any cosmic $\mathrm{X}$-ray emission is negligible.

The background subtraction for each source product (spectrum or profile) is done in two steps. In a first step, we subtract the corresponding product of the blank field observation, obtained using the same energy and spatial selection, normalized by the factor $Q$. From Eqs. (A.1, A.2, A.3, A.4, A.5):

$$
\begin{aligned}
O(x, y, E)-Q T(x, y, E) \equiv & S(x, y, E)+O_{\mathrm{CXB}}(E) \\
& -Q T_{\mathrm{CXB}}(E)
\end{aligned}
$$

and the NXB contribution is thus removed. The remaining CXB component, which can be considered uniform, can thus be estimated, using data outside the source region, where:

$O(x, y, E)-Q T(x, y, E) \equiv O_{\mathrm{CXB}}(E)-Q T_{\mathrm{CX \textrm {B }}}(E)$.

It can then subtracted in a second step.

To illustrate the method, let us consider that we want to estimate the source spectrum in a particular region, Reg:

$S_{\mathrm{Reg}}(E)=\sum_{\operatorname{Reg}} S(x, y, E)$

In a first step, we extract the region spectrum from the observation data set. The spectrum extracted in the same region from the template file is then subtracted, after normalization by the factor $Q$ above. The resulting spectrum is:

$$
\begin{aligned}
I_{\mathrm{Reg}}(E)= & \sum_{\operatorname{Reg}} O(x, y, E)-Q \sum_{\operatorname{Reg}} T(x, y, E) \\
\equiv & S_{\operatorname{Reg}}(E) \\
& +A_{\operatorname{Reg}}\left(O_{\mathrm{CXB}}(E)-Q T_{\mathrm{CXB}}(E)\right)
\end{aligned}
$$

where $A_{\text {Reg }}$ is the surface of the extraction region. We are thus left with a residual $\mathrm{X}$-ray background component, which is the estimate of the difference between the CXB spectrum in the observation and in the template file (multiplied by the normalization factor).

In a second step, we do the same for a region $\mathrm{Reg}^{\prime}$ outside the source. The resulting spectrum is:

$I_{\mathrm{Reg}^{\prime}}(E) \equiv A_{\mathrm{Reg}^{\prime}}\left(O_{\mathrm{CXB}}(E)-Q T_{\mathrm{CXB}}(E)\right)$.

The source spectrum can thus be estimated by subtracting this spectrum to the spectrum obtained in Step 1, after normalization to the size of the extraction region. From Eqs. (A.10) and (A.11) one get:

$I_{\mathrm{Reg}}(E)-\frac{A_{\mathrm{Reg}}}{A_{\mathrm{Reg}^{\prime}}} I_{\mathrm{Reg}^{\prime}}(E) \equiv S_{\mathrm{Reg}}(E)$. 
The same method can be applied to extract the source surface brightness profile, in a given energy band. We extract the surface brightness profile from the observation data set. The surface brightness profile extracted in the same energy band from the template file is then subtracted, after normalization by the factor $Q$ above. The resulting surface brightness profile is an estimate of:

$$
I(r) \equiv S(r)+\left(S_{O_{\mathrm{CXB}}}-Q S_{T_{\mathrm{CXB}}}\right)
$$

where $S(r)$ is the source surface brightness profile and $S_{O_{\mathrm{CXB}}}$ and $S_{T_{\mathrm{CXB}}}$ are the CXB surface brightness in the energy band considered for the observation and template file respectively. The residual CXB contribution can be estimated from the measured profile, $I(r)$, in the region outside the source and subtracted.

\section{References}

Adami, C., Ulmer, M. P., Romer, A. K, et al. 2000, ApJS, 131, 391

Arabadjis, J. S., Bautz, M. W., \& Garmire, G. M. 2002, ApJ, to appear [astro-ph/0109141]

Arnaud, M., \& Evrard, A. E. 1999, MNRAS, 305, 631

Arnaud, M. 2001, in the Proceedings of the XXIth Moriond Astrophysics Meeting, Clusters of galaxies and the high redshift Universe Observed in X-rays (March 2001), ed. D. Neumann, F. Durret, \& J. Tran Thanh Van, to appear

Arnaud, M., Neumann, D. M., Aghanim, A., et al. 2001, A\&A, 365, L80

Arnaud, M., Aghanim, A., \& Neumann, D. M. 2002, A\&A, 389, 1

Bartlett, J., Aghanim, N., Arnaud, M., et al. 2001, in the Proceedings of the XXIth Moriond Astrophysics Meeting, Clusters of galaxies and the high redshift Universe Observed in X-rays (March 2001), ed. D. Neumann, F. Durret, \& J. Tran Thanh Van, to appear

Borgani, S., Governato, F., Wadsley, J., et al. 2001a, ApJ, 559, L71

Borgani, S., Rosati, P., Tozzi, P., et al. 2001b, ApJ, 561, 13

Bryan, G. L., \& Norman, M. L. 1998, ApJ, 495, 80

De Grandi, S., \& Molendi, S. 2001, ApJ, 551, 153

Dickey, J. M., \& Lockman, F. J. 1990, ARA\&A, 28, 215

Eke, V. R., Navarro, J. F., \& Frenk, C. S. 1998, ApJ, 503, 569

Elbaz, D., Arnaud, M., \& Böhringer, H. 1995, A\&A, 293, 337

Evrard, A. E., \& Henry, J. P. 1991, ApJ, 383, 95

Evrard, A. E., Metzler, C. A., \& Navarro, J. F. 1996, ApJ, 469, 494

Fairley, B. W., Jones, L. R., Scharf, C., et al. 2000, MNRAS, 315, 669

Ghizzardi, S. 2001, EPIC-MCT-TN-011 (XMM-SOC-CAL-TN0022)
Gioia, I. M., Maccacaro, T., Schild, R. E., et al. 1990, ApJS, 72, 567

Gioia, I. 2000, in Constructing the Universe with Clusters of Galaxies, IAP 2000 meeting, Paris, France, July 2000, ed. Florence Durret \& Daniel Gerbal, cucg.confE8

Griffiths, R. G., Briel, U., Dadida, M., et al. 2002, in Proc. of the New Vision of the X-ray Universe in the XMM-Newton and Chandra Era conference, to appear

Griffiths, G., \& Saxton, R. 2002, in preparation

Holden, B. P., Stanford, S. A., Rosati, P. et al. 2001, ApJ, 122, 629

Kaastra, J. S. 1992, An X-ray spectral Code for Optically Thin Plasmas, Internal SRON-Leiden Report, updated version 2.0

Kaiser, N. 1991, ApJ, 383, 104

Lumb, D. 2002, XMM-SOC-CAL-TN-0016

Liedahl, D. A., Osterheld, A. L., \& Goldstein, W. H. 1995, ApJ, 438, 115

Lloyd-Davies, E. J., Ponman, T. J., \& Cannon, D. B. 2000, MNRAS, 315,689

Majerowicz, S., Neumann, D. M., \& Reiprich, T. H. 2002, A\&A, submitted [astro-ph/0202347]

Mewe, R., Gronenschild, E. H. B. M., \& van den Oord, G. H. J. 1985, A\&AS, 62, 197

Mewe, R., Lemen, J. R., \& van den Oord, G. H. J. 1986, A\&AS, 65, 511

Mohr, J. J., Mathiesen, B., \& Evrard, A. E. 1999, ApJ, 517, 627

Mohr, J. J., Reese, J. J., Ellingson, E., et al. 2000, ApJ, 544, 109

Navarro, J. F., Frenk, C. S., \& White, S. D. M. 1997, ApJ, 490, 493

Neumann, D. M., \& Arnaud, M. 1999, A\&A, 348, 711

Neumann, D. M., \& Arnaud, M. 2000, ApJ, 542, 35

Neumann, D. M., \& Arnaud, M. 2001, A\&A, 373, L33

Oukbir, J., \& Blanchard, A. 1997, A\&A, 317, 1

Pearce, F. R., Thomas, P. A., Couchman, H. M. P., et al. 2000, MNRAS, 317, 1029

Pen, U. 1997, New Astron., 2, 309

Ponman, T. J., Cannon, D. B., \& Navarro, J. F. 1999, Nature, 397, 135

Reichart, D. E., Castander, F. J., \& Nichol, R. C. 1999, ApJ, 516, 1

Rines, K., Forman, W., Pen, U., et al. 1999, ApJ, 517, 70

Romer, A. K., Nichol, R. C., Holden, B. P., et al. 2000, ApJS, 126, 209

Sadat, R., Blanchard, A., \& Oukbir, J. 1998, A\&A, 329, 21

Sarazin, C. 1986, Rev. Mod. Phys., 58, 1

Saxton, R. 2002, XMM-SOC-CAL-TN-0023

Snowden, S. 2002, in Proc. of the New Vision of the X-ray Universe in the XMM-Newton and Chandra Era conference, to appear

Strüder, L., Briel, U., Dennerl, K., et al. 2001, A\&A, 365, L18

Tozzi, P., \& Norman, C. 2001, ApJ, 546, 63

Turner, M. J. L., Abbey, A., Arnaud, M., et al. 2001, A\&A, 365, L27 